\title{
Aplicação da Metodologia de Superfície de Resposta para Otimização do Processo de Solda a Ponto no Aço Galvanizado AISI 1006
}

\author{
Fernando Ferraz Amaral ${ }^{1}$, Fabrício Alves de Almeida ${ }^{1}$, Sebastião Carlos Costa ${ }^{1}$, Rafael Coradi Leme ${ }^{1}$, Anderson Paulo de Paiva ${ }^{1}$ \\ 1 Universidade Federal de Itajubá - UNIFEI, Instituto de Engenharia de Produção e Gestão, Itajubá, MG, Brasil.
}

Recebido: 08 Mar., 2018

Aceito: 16 Maio, 2018

E-mail: fferrazamaral@yahoo.com.br (FFA)
Resumo: A soldagem a ponto por resistência elétrica possui um papel importante na fabricação de diversos produtos da indústria. Apesar de sua ampla aplicabilidade, este processo está sujeito a apresentar certa inconsistência na qualidade devido a variáveis controláveis e não controláveis. Desta forma, este estudo busca aplicar a metodologia de superfície de resposta para a obtenção de valores otimizados de fatores controláveis do processo, tais como corrente de soldagem, tempo de soldagem e força do eletrodo. Verificou-se o comportamento das curvas do deslocamento do eletrodo, a fim de observar o efeito da queima do revestimento das chapas de aço carbono galvanizadas. Avaliou-se também o teste de cisalhamento para verificar a deformação na força máxima de ruptura dos corpos de prova. Os resultados da aplicação mostraram que parâmetros elevados de corrente e parâmetros reduzidos dos tempos de ciclo proporcionaram maiores níveis de força e valores desejáveis de indentação. Além disso, foi possível verificar através dos experimentos que os critérios de qualidade do ponto de solda apresentam correlação com as características das curvas de deslocamento do eletrodo, proporcionando uma maneira eficaz para avaliar a qualidade do processo de solda a ponto a partir de testes não destrutivos.

Palavras-chave: Solda a ponto por resistência elétrica; Metodologia de superfície de resposta; Otimização; Aço galvanizado; Deslocamento do eletrodo.

\section{Application of the Response Surface Methodology for Optimization of the Resistance Spot Welding Process in AISI 1006 Galvanized Steel}

\begin{abstract}
The resistance spot welding has a great importance in the manufacture of several industrial products. Despite its wide applicability, this process is subject to certain quality inconsistency due to controllable and non-controllable variables. Thus, this study seeks to apply the response surface methodology to obtain optimized values of controllable process factors, such as welding current, welding time and electrode strength. The behavior of the electrode displacement curves has been verified in order to observe the burning effect of the coating of the galvanized carbon steel plates. It was also evaluated the shear test to verify the tensile-shear strength of the specimens. The results of the application showed that high current parameters and reduced cycle time parameters provided higher levels of strength and desirable indentation values. In addition, it was possible to verify through the experiments that the quality criteria of the weld point correlate with the characteristics of the displacement curves of the electrode, providing an efficient way to evaluate the quality of the process of welding to point from nondestructive tests.
\end{abstract}

Key-words: Resistance spot welding; Response surface methodology; Optimization; Galvanized steels; Electrode displacement.

\section{Introdução}

A soldagem a ponto por resistência elétrica (RSW-Resistance Spot Welding) possui grande importância na fabricação de diversos produtos da indústria, sendo um dos processos mais utilizados em produções seriadas e abrangendo muitos segmentos industriais como automobilísticas, eletrônicas, nucleares, tubulações, equipamentos ferroviários, aeroespacial, entre outras [1,2]. A facilidade de operação e de automação, rapidez, realização de vários pontos de solda sem que sejam necessários grandes ajustes dos parâmetros, e o baixo investimento, são os principais fatores que levam a grande utilização deste processo abrindo possibilidades para a obtenção de produtos de alta qualidade [3]. 
Os aços galvanizados são materiais largamente usados no setor automobilístico entre outros, e sua soldabilidade através do processo RSW é alvo de constantes pesquisas. A presença nestes materiais de uma fina camada superficial de zinco, faz com que, para que a soldagem efetivamente se concretize, haja a necessidade da sua remoção durante o processo de soldagem no intuito de obter uma ligação adequada, garantindo a qualidade final do ponto soldado. Entretanto, é necessário entender os aspectos do comportamento do processo, além da relação com o ajuste dos parâmetros do processo de soldagem a ponto. Para isto, deve-se utilizar sensores de deslocamento para avaliar a movimentação dos eletrodos durante a aplicação de calor sobre as peças [4-6].

Considerando o fato de que o processo RSW apresenta como característica principal a sua rapidez de execução, conhecer a influência dos parâmetros do processo é condição inicial para melhorar os ajustes e objetivos pré-definidos. Deste modo, para que se tenha um ajuste preciso destes parâmetros, se faz necessário conhecer modelagens matemáticas que associem as influências em determinadas características desejáveis da solda, garantindo a versatilidade do processo em ambientes industriais. Para minimizar os custos experimentais é necessário utilizar estratégias como o delineamento de experimentos (DOE - Design of Experiments), que, segundo Almeida et al. [7], é uma técnica estatística capaz de modelar e otimizar experimentos. Segundo Naves et al. [8], metodologia de superfície de resposta (RSM - Response Surface Methodology) é um tipo de DOE amplamente utilizado em vários seguimentos, como nos trabalhos de Tak et al. [9], Pi et al. [10], Sheydaei et al. [11], Anouzla et al. [12]. Entre todos os projetos de RSM, existe um arranjo específico conhecido como o arranjo composto central (CCD - Central Composite Design). O CCD é o projeto mais utilizado para experimentos, apresentando maiores vantagens que outros tipos de design, sendo capaz de gerar modelos quadráticos completos [7,13]. Desta maneira, tem-se que determinar as condições ótimas de operação para um sistema ou o conhecer a região do espaço amostral, torna-se possível fornecer informações relevantes para aplicação dos métodos estatísticos de análises que são amplamente utilizadas em estudos envolvendo o processo RSW [14-19].

Um estudo experimental foi realizado para modelar as respostas de interesse (relativas ao processo RSW) onde um arranjo do tipo composto central, derivado da RSM, foi criado para modelar as quatro variáveis de processo (Corrente, tempo, pressão e pré-aquecimento). Deste modo, tem-se que o objetivo deste estudo é aplicar a Metodologia de Superfície de Resposta para obtenção dos níveis mais adequados dos parâmetros de corrente de solda, tempo de solda, pressão dos eletrodos e pré-aquecimento para verificar as respostas de qualidade: resistência final e indentação, obtidas na soldagem a ponto em chapas de aço galvanizado AISI 1006. Além disso, o estudo propõe uma análise das curvas de deslocamento do eletrodo geradas durante a soldagem, correlacionando-as com a qualidade dos pontos de solda.

Este manuscrito está organizado da seguinte forma: A seção 2 apresenta uma abordagem sobre a qualidade da solda a ponto em aços galvanizados. Subsequente, na seção 3 tem-se uma breve revisão da técnica utilizada no estudo, sendo esta a RSM. Na seção 4 é apresentado o procedimento experimental. A aplicação do método, detalhando o projeto, etapas, resultados e os experimentos de confirmação, são descritos na seção 5 . Finalmente, a seção 6 descreve as conclusões encontradas neste estudo.

\section{Qualidade da Solda a Ponto por Resistência Elétrica em Aços Galvanizados}

Basicamente, para gerar uma solda de qualidade, deve-se obter um tamanho apropriado do ponto de solda, principalmente na direção que define a penetração da solda. Uma penetração aceitável deve estar entre $60 \%$ e $70 \%$ da espessura das duas chapas soldadas [20]. Além da aparência, um ponto de solda de qualidade deve apresentar uma resistência significativa. Estes são requisitos fundamentais para os padrões industriais.

De acordo com Gedeon e Eagar [4], em seu estudo verificou-se que durante o processo de soldagem o comportamento padrão do deslocamento dos eletrodos apresenta seis fases subsequentes, como ilustradas na Figura 1. Na fase 1 observa-se a queda no deslocamento devido ao achatamento das asperezas na superfície do material perante a aplicação da pré-pressão. Na fase 2 ocorre uma expansão térmica da camada protetora de zinco, além da expansão do material base, entretanto, a presença da camada de zinco nas chapas faz com que as mesmas se afastem gradual e lentamente pela pressão dos eletrodos, gerando uma leve inclinação da curva comparada com a fase 4 onde predomina a expansão térmica. $\mathrm{Na}$ fase 3 , a camada de zinco se torna líquida, sendo afastada dos eletrodos mais rapidamente que nos estágios anteriores. $O$ deslocamento nesta fase é maior que a expansão térmica, resultando em pequeno decréscimo, com pequena inclinação (pequeno slope). Na fase 5 observa-se que 
Aplicação da Metodologia de Superfície de Resposta para Otimização do Processo de Solda a Ponto no Aço Galvanizado AISI 1006

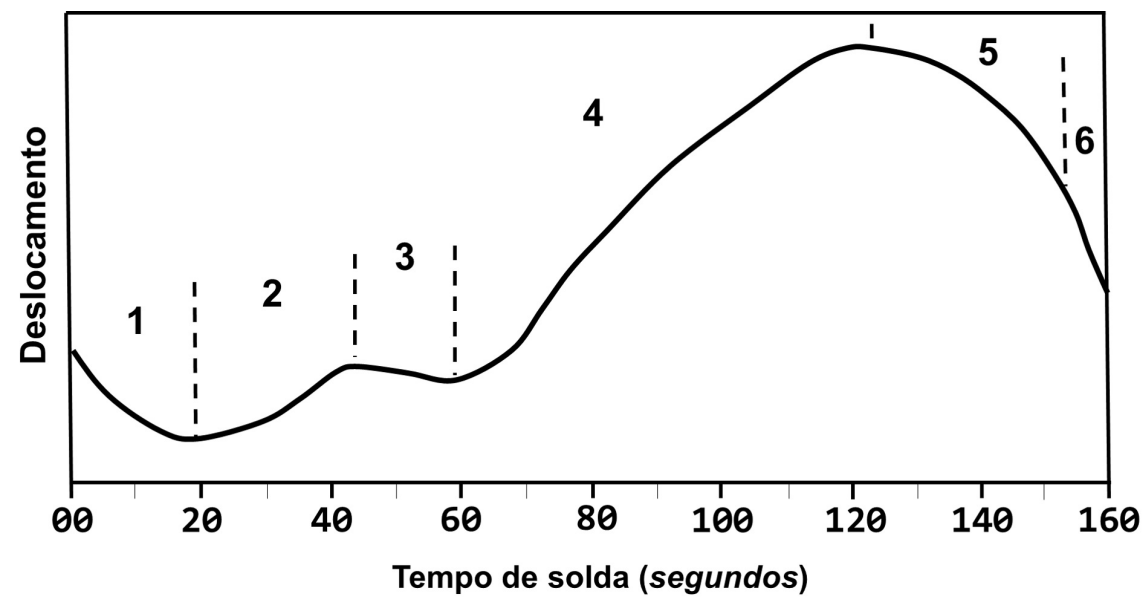

Figura 1. Fases do deslocamento típicas em aços galvanizados. Adaptado de Gedeon e Eagar [4].

o deslocamento começa a decrescer, devido ao aço se tornar macio pelo alto aquecimento. Ainda nesta fase, a corrente deve ser desligada para que a indentação não se torne proeminente. Na fase 6 , caso a corrente se situe num nível muito alto, com um tempo de solda muito longo, a expulsão de material pode ocorrer.

A indentação, presente na região 5 , é fator comum nos processos de solda a ponto. Isto pode ocorrer quando a resistência do material em contato com o eletrodo diminui à medida que a fusão avança, permitindo, deste modo, que a face do eletrodo se movimente para dentro da superfície da peça. O controle da indentação é bastante utilizado nas indústrias como uma forma de controlar a qualidade dos pontos de solda. Conforme demostrado em Aslanlar [5], uma indentação em torno de $20 \%$ da espessura mínima da chapa soldada é um indicativo de boa qualidade da solda em aços galvanizados. Porém, este limite pode oscilar de acordo com os critérios de aparência e resistência exigidos.

Para verificar a resistência dos pontos de solda no que concerne as propriedades mecânicas, o teste por tensão cisalhante é amplamente utilizado, como em estudos recentes de: Manladan et al. [21]; Shan et al. [22], Chen et al. [23], Martín et al. [24] e Feng et al. [25]. Este consiste na aplicação de forças de separação nas duas chapas soldadas. Estas forças são opostas e agem em uma direção paralela à superfície da peça, conforme ilustrado na Figura 2. De forma geral, o modo de falha característico pós solda é utilizado como parâmetro importante na verificação de performance das condições de solda impostas ao material e podem ocorrer sob a forma de falha interfacial caracterizando uma baixa resistência da junção; ou por arrancamento caracterizando uma boa resistência ou por com rasgamento da chapa ao redor da solda característico de soldas de alta resistência [6,26]. A caracterização deste modo de falha está relacionado as condições de ajuste do parâmetros e o comportamento microestrutural do material.

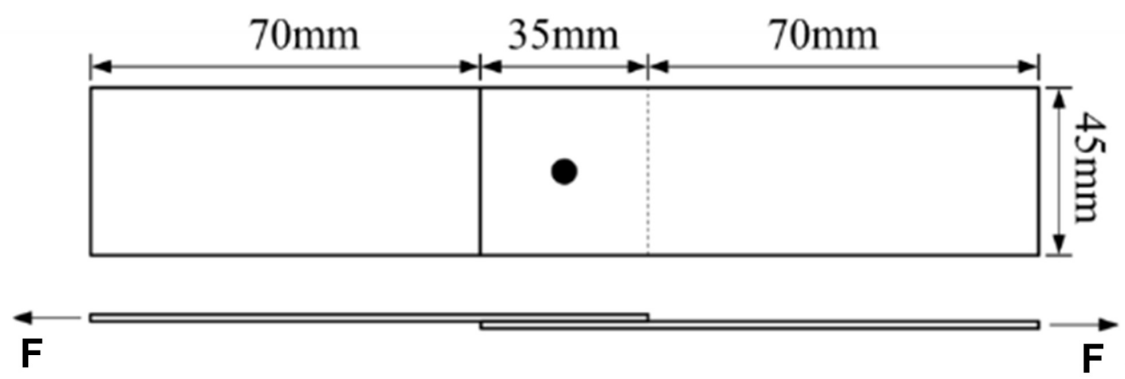

Figura 2. Representação do ensaio de cisalhamento. Adaptado de Wan et al. [27]. 


\section{Metodologia de Superfície de Resposta}

A Metodologia de Superfície de Resposta é uma coleção de técnicas matemáticas e estatísticas que são utilizadas para modelar e analisar problemas nos quais a resposta de interesse é influenciada por diversas variáveis, nos quais a resposta deva alcançar um valor ótimo [13], caracterizando-se como uma metodologia prática, econômica e de fácil implementação [28]. A análise de uma superfície de resposta é feita em termos de uma superfície ajustada. Se tal superfície é adequada, sua análise será aproximadamente equivalente a análise da superfície real. Comumente, um determinado ponto da superfície de resposta que apresenta-se distante do ponto de ótimo, a superfície apresenta uma curvatura suave.

De acordo com Almeida et al. [7], o polinômio de segunda ordem representa significativamente os problemas para a superfície de resposta (Equação 1).

$$
Y=\beta_{0}+\sum_{i=1}^{k} \beta_{i} x_{i}+\sum_{i=1}^{k} \beta_{i i} x_{i}^{2}+\sum_{i<j} \sum \beta_{i j} x_{i} x_{j}+\varepsilon
$$

onde $Y$ é a resposta de interesse, $k$ o número de variáveis independentes, $\beta$ os coeficientes estimados, $x$ os parâmetros e $\varepsilon$ o termo de erro associado. Neste estágio, deve-se localizar o ponto ótimo. Portanto, o objetivo da RSM é determinar as condições ótimas de operação para um sistema ou determinar a região do espaço na qual as necessidades operacionais sejam satisfeitas.

O projeto quadrático denominado Arranjo Composto Central (CCD - Central Composite Design), é formado por três grupos distintos de elementos experimentais: um fatorial completo ou um fatorial fracionário, um conjunto de pontos centrais e, adicionalmente, um grupo de níveis extras denominados pontos axiais. Se a distância entre o centro do arranjo e ponto fatorial $(+1 ;-1)$ for aproximadamente 1 (em módulo), a distância do centro ao ponto axial será maior que a unidade [13]. O arranjo do tipo CCD é recomendado quando o planejamento fatorial necessita de experimentação sequencial. Isto porque, estes projetos podem incorporar informações de um experimento fatorial apropriadamente planejado. O número de pontos axiais em um CCD é igual ao dobro do número de fatores e representam os valores extremos de cada fator. Em função de sua localização, podem ser circunscritos, inscritos ou de face centrada. O Circunscrito (CCC) é o CCD original. Nele, os pontos axiais estão a uma distância a do centro, baseado nas propriedades desejadas do projeto.

\section{Procedimentos Experimentais}

Os experimentos foram realizados em um laboratório de soldagem utilizando uma máquina de solda a ponto estacionária, marca $\operatorname{Preso}^{\circledR}$, modelo TWPRV50, com acionamento pneumático e potência nominal de 50 kVA. Para monitoramento do deslocamento, foi utilizado um sensor de posição LVDT acoplado a estrutura do acionamento pneumático do eletrodo, o qual permitiu avaliar o deslocamento do eletrodo durante a execução do processo de soldagem. A aquisição dos dados foi procedida através de um condicionador digital modelo SD20 com saída USB para aplicação com sensores LVDT, além de um conversor A/D 24-bits para linearização digital de todo campo de medição. As medições foram posteriormente convertidas para o software Excel ${ }^{\circledR}$. Os detalhes do banco de ensaios podem ser observados na Figura 3.
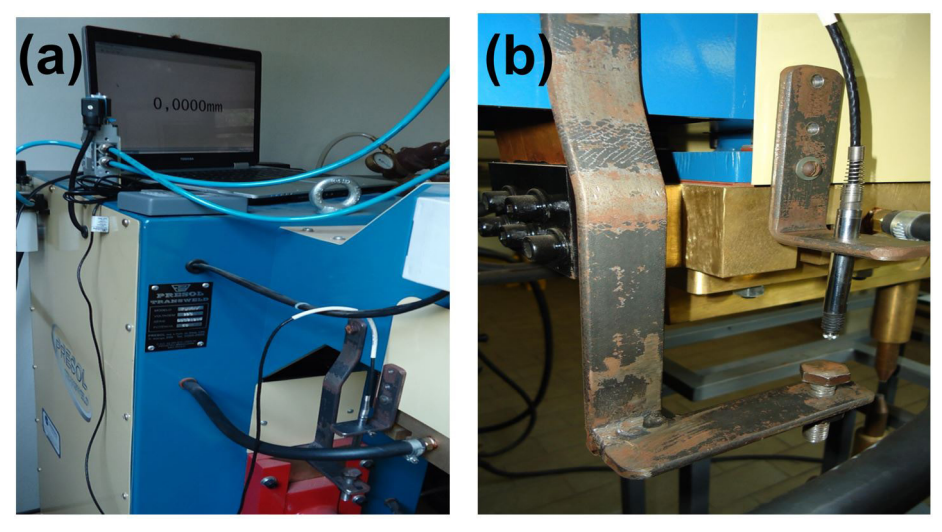

Figura 3. Componentes da máquina de solda a ponto. (a) Equipamento com sensor de deslocamento; (b) detalhes do sensor de deslocamento. 
O material de base utilizado foi o aço carbono eletro galvanizada AISI 1006, com composição de $0,08 \%$ C; $0,45 \% \mathrm{Mn} ; 0,03 \% \mathrm{P} ; 0,03 \% \mathrm{~S} ; 0,005 \% \mathrm{Al} ; 42,5-85 \mathrm{~g} / \mathrm{m}^{2} \mathrm{Zn}$ e $1 \mathrm{~mm}$ de espessura. Os corpos de prova para execução dos testes foram dimensionados de acordo com a norma AWS [29], descritos na Figura 4. Após a soldagem, os corpos de prova foram analisados e, posteriormente, submetidos ao teste de cisalhamento.

Como respostas, foram avaliadas após a soldagem:

- Indentação máxima do ponto de solda. Medição obtida por meio de um apalpador analógico Mitutoyo com resolução de $0,01 \mathrm{~mm}$;

- $\quad$ Força máxima de cisalhamento suportada pelo ponto de solda. Testes destrutivos realizados utilizando uma máquina de tração $E M I C^{\circledR} D L 2000$;

- $\quad$ Caracterização do modo de falha das chapas soldadas após teste de cisalhamento. Avaliação pela análise do modo de fratura do ponto de solda, sendo os mesmos classificados como interfacial, arrancamento do ponto ou rasgamento da chapa, conforme padronização mostrada na Figura 5.

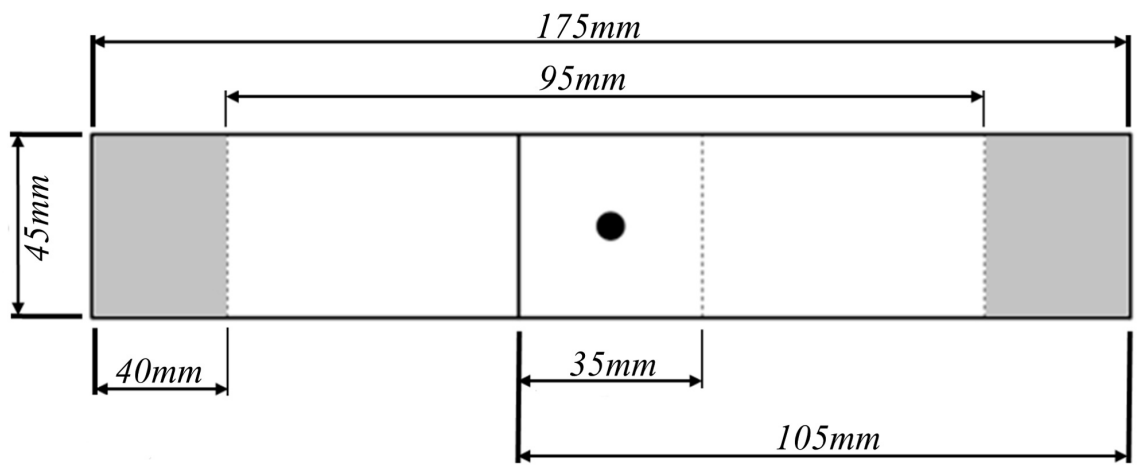

Figura 4. Especificações dimensionais dos corpos de prova. Adaptado de AWS [29].
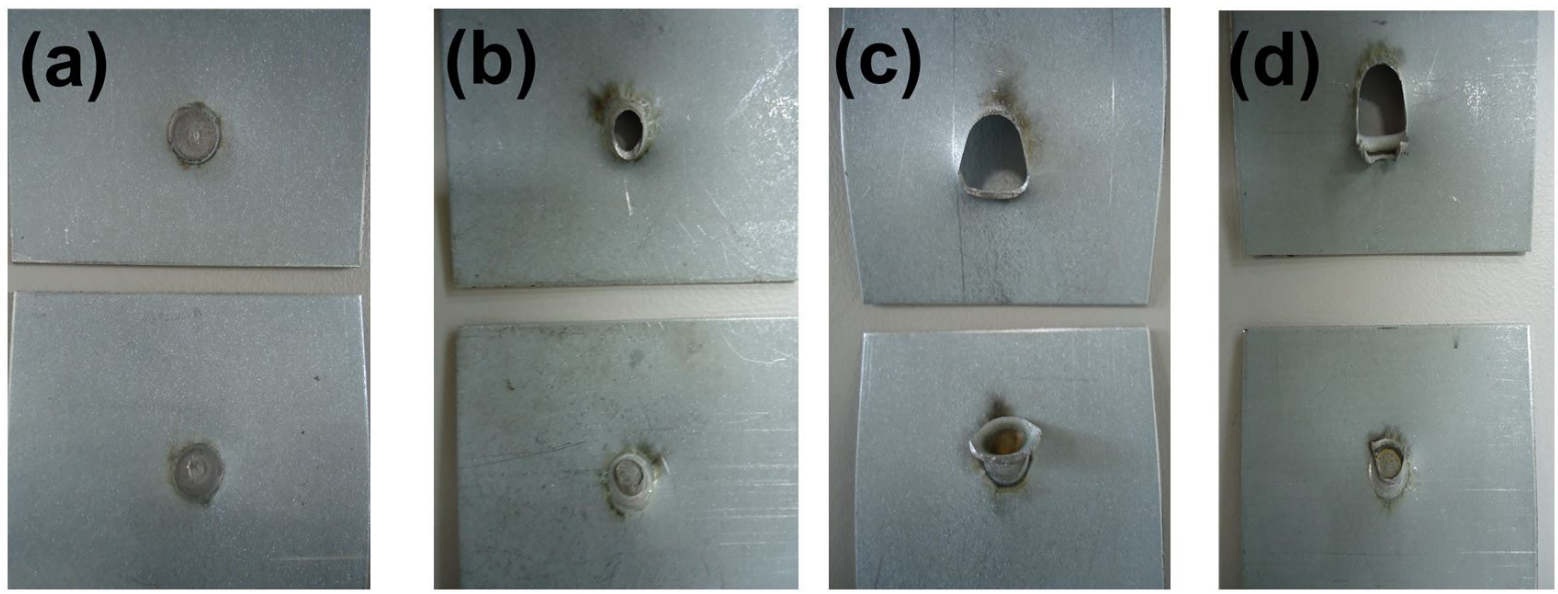

Figura 5. Modo de falha padronizado: (a) interfacial; (b) arrancamento do ponto; (c) rasgamento; e (d) arrancamento do ponto com rasgamento da chapa.

As análises estatísticas foram conduzidas através do software Minitab18 ${ }^{\circledR}$, e a otimização das respostas foi gerada através da ferramenta Solver ${ }^{\circledR}$ do software Microsoft Excel ${ }^{\circledR}$.

O processo RSW é caracterizado por um grande número de parâmetros ajustáveis, entretanto testes preliminares realizados mostraram que a corrente de solda, tempo de solda, e pressão dos eletrodos são os parâmetros que exercem maior influência na qualidade dos pontos de solda. Estes parâmetros de controle estão presente em 
diversos trabalhos que utilizam a RSW, tais como: Wan et al. [30], Zhang et al. [31], Pouranvari [32] e Ighodaro et al. [33]. Entretanto, para chapas de aço galvanizado, foi possível verificar que o pré-aquecimento (composto por uma combinação de tempo e corrente de soldagem) também mostrou-se eficaz para aumentar a resistência dos pontos de solda, contribuindo para a eliminação da camada superficial de zinco. Deste modo, optou-se por incluir a variável de pré-aquecimento na análise do processo. Sendo assim, os experimentos foram conduzidos fixando o parâmetro de pré-aquecimento com uma corrente de $50 \%(3 \mathrm{kA})$ e variando somente o tempo de ciclo.

Diante aos quatro parâmetros principais, os testes foram realizados com os níveis mínimos que garantissem a não ocorrência do modo de falha interfacial no ponto de solda (Figura 5a). Deste modo, os níveis foram estabelecidos de acordo com os limites operacionais do objeto de estudo, conforme descritos na Tabela 1.0 arranjo idealizado foi composto quatro fatores de dois níveis $\left(2^{k}=2^{4}=16\right)$, oito pontos axiais $(2 \mathrm{k}=8)$ e cinco pontos centrais totalizando 29 combinações. Os experimentos foram executados de forma aleatória com o objetivo de evitar tendências.

Tabela 1. Definição dos níveis para análise experimental.

\begin{tabular}{ccccccc}
\hline \multirow{2}{*}{ Fatores controláveis } & Unidade & \multicolumn{5}{c}{ Níveis } \\
\cline { 3 - 7 } & & $-\mathbf{1 . 6 8 2}$ & $-\mathbf{1}$ & $\mathbf{0}$ & $\mathbf{1}$ & $\mathbf{1 . 6 8 2}$ \\
Corrente de soldagem (A) & $\% k A$ & 85 & 86 & 87 & 88 & 89 \\
Tempo de soldagem (B) & Ciclos & 9 & 10 & 11 & 12 & 13 \\
Pressão dos eletrodos (C) & Bar & 3,5 & 4 & 4,5 & 5 & 5,5 \\
Pré-aquecimento (D) & Ciclos/\%kA & N/A & $0 / 0$ & $3 c / 50(3)$ & $6 c / 50(3)$ & $9 c / 50(3)$ \\
\hline
\end{tabular}

\section{Resultados e Análises}

A seqüência de experimentos e os resultados das respostas de força máxima de cisalhamento, indentação máxima entre as duas faces do ponto e o modo de falha podem ser observados na Tabela 2.

A Tabela 3 apresenta a análise de variância (ANOVA) para a força máxima de cisalhamento e indentação, onde observa-se que para ambas as respostas, os coeficientes de correlação e ajuste dos modelos se mostraram bastante adequados.

Com relação as respostas de força de cisalhamento e indentação máxima, chegou-se ao modelo reduzido, descrito na Tabela 3, após eliminar os fatores não significativos considerando um nível de significância de $5 \%$ (valor $p<0,05)$. Observa-se que os parâmetros corrente de soldagem $(A)$, tempo de soldagem $(B)$ e pré-aquecimento $(D)$, assim como o quadrado de pressão dos eletrodos $\left(C^{2}\right)$ e a interação entre corrente de soldagem e pré-aquecimento $(A x D)$, mostraram exercer um impacto significativo na resposta de força máxima de cisalhamento. Apesar dos demais termos não terem grau significativo, o modelo final reduzido considerando os coeficientes lineares, quadráticos e interações, apresentou maior porcentagem de explicação comparado ao modelo completo, $R_{a d j}^{2}$ de $92,20 \%$ e $R_{a d j}^{2}$ de $91,83 \%$, respectivamente. Além disso, o modelo final apresentou-se com ajuste adequado (Lack-of-Fit de 0,319). Com relação a indentação máxima, todos os parâmetros, o quadrado do tempo de soldagem $\left(B^{2}\right)$ e o quadrado do pré-aquecimento $\left(D^{2}\right)$ mostraram impacto direto na indentação máxima. O modelo reduzido também apresentou um elevado nível de explicação $\left(R^{2}{ }_{a d j}\right.$ de $93,8 \%$ ) e ajuste adequado (Lack-of-Fit de 0,268).

A análise de resíduos gerados para as respostas de força máxima de cisalhamento e indentação máxima são mostrados na Figura 6. Pode-se notar que, para ambas as respostas, as amostras apresentam uma distribuição normal e nenhuma observação está a mais de 3 desvios padrões da média das amostras.

A partir das Figuras 7 e 8, pode-se observar as superfícies de resposta para a força máxima de cisalhamento e a indentação máxima, respectivamente. Ambas as respostas apresentam curvaturas nas superfícies, onde as curvas para força máxima apresentam um perfil côncavo, favorável para localizar um ponto de máximo, e curvas para a indentação máxima apresentaram um perfil convexo, favorável para localizar um ponto mínimo. Considerando que, para se conseguir um ponto de solda de qualidade, deve-se maximizar a resistência e manter a indentação dentro de um determinado nível (por volta de $20 \%$ ), tem-se os modelos propostos, representados pelas Equações 2 e 3 , 
Aplicação da Metodologia de Superfície de Resposta para Otimização do Processo de Solda a Ponto no Aço Galvanizado AISI 1006

Tabela 2. Matriz e resultado dos experimentos.

\begin{tabular}{|c|c|c|c|c|c|c|c|}
\hline \multirow[b]{2}{*}{ Experimentos } & \multicolumn{4}{|c|}{ Matriz experimental } & \multicolumn{3}{|c|}{ Respostas } \\
\hline & Corrente & Tempo & Pressão & Pré-aq & $\begin{array}{l}\text { Identação } \\
\text { Máxima }\end{array}$ & $\begin{array}{l}\text { Força } \\
\text { Máxima }\end{array}$ & Modo de falha \\
\hline 1 & 86 & 12 & 4 & 0 & 0,13 & 4184 & $\begin{array}{c}\text { arrancamento / } \\
\text { rasgamento }\end{array}$ \\
\hline 2 & 88 & 12 & 4 & 0 & 0,18 & 4312 & rasgamento \\
\hline 3 & 88 & 12 & 5 & 1 & 0,27 & 4384 & $\begin{array}{c}\text { arrancamento / } \\
\text { rasgamento }\end{array}$ \\
\hline 4 & 88 & 10 & 4 & 1 & 0,17 & 4206 & rasgamento \\
\hline 5 & 87 & 11 & 3,5 & 0,5 & 0,16 & 4130 & rasgamento \\
\hline 6 & 86 & 10 & 4 & 1 & 0,13 & 4118 & rasgamento \\
\hline 7 & 88 & 10 & 5 & 0 & 0,18 & 3916 & arrancamento \\
\hline 8 & 88 & 10 & 5 & 1 & 0,2 & 4269 & rasgamento \\
\hline 9 & 87 & 11 & 4,5 & 1,5 & 0,19 & 4510 & $\begin{array}{c}\text { Arrancamento / } \\
\text { rasgamento }\end{array}$ \\
\hline 10 & 86 & 10 & 4 & 0 & 0,1 & 3904 & arrancamento \\
\hline 11 & 87 & 13 & 4,5 & 0,5 & 0,28 & 4450 & $\begin{array}{c}\text { arrancamento / } \\
\text { rasgamento }\end{array}$ \\
\hline 12 & 87 & 11 & 4,5 & 0,5 & 0,17 & 4250 & $\begin{array}{c}\text { arrancamento / } \\
\text { rasgamento }\end{array}$ \\
\hline 13 & 87 & 11 & 5,5 & 0,5 & 0,21 & 4127 & arrancamento \\
\hline 14 & 87 & 9 & 4,5 & 0,5 & 0,15 & 3857 & arrancamento \\
\hline 15 & 86 & 10 & 5 & 1 & 0,17 & 4024 & arrancamento \\
\hline 16 & 87 & 11 & 4,5 & $-0,5$ & $*$ & $*$ & $*$ \\
\hline 17 & 87 & 11 & 4,5 & 0,5 & 0,18 & 4202 & rasgamento \\
\hline 18 & 88 & 12 & 4 & 1 & 0,24 & 4490 & rasgamento \\
\hline 19 & 86 & 12 & 5 & 1 & 0,26 & 4197 & $\begin{array}{c}\text { arrancamento / } \\
\text { rasgamento }\end{array}$ \\
\hline 20 & 86 & 12 & 5 & 0 & 0,21 & 4177 & $\begin{array}{c}\text { arrancamento / } \\
\text { rasgamento }\end{array}$ \\
\hline 21 & 89 & 11 & 4,5 & 0,5 & 0,23 & 4279 & $\begin{array}{c}\text { arrancamento / } \\
\text { rasgamento }\end{array}$ \\
\hline 22 & 85 & 11 & 4,5 & 0,5 & 0,16 & 4028 & rasgamento \\
\hline 23 & 87 & 11 & 4,5 & 0,5 & 0,17 & 4226 & $\begin{array}{c}\text { arrancamento / } \\
\text { rasgamento }\end{array}$ \\
\hline 24 & 87 & 11 & 4,5 & 0,5 & 0,19 & 4167 & $\begin{array}{c}\text { arrancamento / } \\
\text { rasgamento }\end{array}$ \\
\hline 25 & 87 & 11 & 4,5 & 0,5 & 0,18 & 4159 & rasgamento \\
\hline 26 & 88 & 10 & 4 & 0 & 0,12 & 3947 & $\begin{array}{c}\text { arrancamento / } \\
\text { rasgamento }\end{array}$ \\
\hline 27 & 88 & 12 & 5 & 0 & 0,24 & 4222 & rasgamento \\
\hline 28 & 86 & 12 & 4 & 1 & 0,19 & 4334 & $\begin{array}{c}\text { arrancamento / } \\
\text { rasgamento }\end{array}$ \\
\hline 29 & 86 & 10 & 5 & 0 & 0,16 & 4001 & arrancamento \\
\hline
\end{tabular}

*Resultado fora do limite operacional do equipamento.

respectivamente. As respostas de força de cisalhamento e indentação apresentam um nível de correlação médio, com valor de Pearson de 0,657 ( $p$-value =0,000), sendo, assim, necessário otimizar as duas respostas de qualidade de forma única. É possível verificar na Figura 9 uma faixa possível de ser coberta com valores de indentação máxima variando entre $0,15 \mathrm{~mm}$ a 0,20 $\mathrm{mm}$, dentro de uma faixa de força máxima de cisalhamento de $4200 \mathrm{~N}$ a $4400 \mathrm{~N}$.

Partindo do pressuposto de que existem limites permissíveis de indentação que podem variar de acordo com a aplicação, um modelo de otimização foi gerado através do Solver ${ }^{\circledR}$ do Excel objetivando maximizar a força máxima de cisalhamento com uma restrição de indentação máxima de $20 \%$, limite comumente utilizado na indústria. As Equações 2 e 3 representam o resumo do modelo proposto. 
Tabela 3. Efeitos principais dos modelos quadráticos e das interações para a força máxima de cisalhamento e indentação máxima.

\begin{tabular}{|c|c|c|c|c|c|c|c|c|}
\hline & \multicolumn{4}{|c|}{ Força } & \multicolumn{4}{|c|}{ Indentação } \\
\hline & $\begin{array}{c}\text { Modelo } \\
\text { completo }\end{array}$ & $p$-value & $\begin{array}{l}\text { Modelo } \\
\text { reduzido }\end{array}$ & $p$-value & $\begin{array}{c}\text { Modelo } \\
\text { completo }\end{array}$ & $p$-value & $\begin{array}{l}\text { Modelo } \\
\text { reduzido }\end{array}$ & $p$-value \\
\hline Coeficiente & 4200,80 & & 4200,80 & & 0,1780 & & 0,1798 & \\
\hline$I(A)$ & 54,54 & 0,000 & 54,54 & 0,000 & 0,0163 & 0,000 & 0,0163 & 0,000 \\
\hline$t(B)$ & 129,21 & 0,000 & 129,21 & 0,000 & 0,0313 & 0,000 & 0,0313 & 0,000 \\
\hline Pres (C) & $-12,96$ & 0,215 & $-12,96$ & 0,202 & 0,0221 & 0,000 & 0,0221 & 0,000 \\
\hline Preaq (D) & 88,42 & 0,000 & 88,42 & 0,000 & 0,0197 & 0,000 & 0,0195 & 0,000 \\
\hline$P^{2}\left(A^{2}\right)$ & $-15,30$ & 0,138 & $-15,30$ & 0,127 & 0,0040 & 0,117 & 0,0037 & 0,107 \\
\hline$t^{2}\left(B^{2}\right)$ & $-15,30$ & 0,138 & $-15,30$ & 0,127 & 0,0090 & 0,002 & 0,0087 & 0,001 \\
\hline $\operatorname{Pres}^{2}\left(C^{2}\right)$ & $-21,55$ & 0,044 & $-21,55$ & 0,038 & 0,0015 & 0,546 & * & * \\
\hline Preaq $^{2}\left(D^{2}\right)$ & 26,13 & 0,054 & 26,13 & 0,047 & $-0,0074$ & 0,028 & $-0,0075$ & 0,016 \\
\hline$I^{*} t\left(A^{*} B\right)$ & 14,06 & 0,268 & * & * & 0,0019 & 0,537 & * & * \\
\hline$I^{*}$ press $\left(A^{*} C\right)$ & $-1,44$ & 0,908 & * & * & $-0,0044$ & 0,163 & $-0,0044$ & 0,131 \\
\hline$I^{*}$ Preag $\left(A^{*} D\right)$ & 34,06 & 0,015 & 34,06 & 0,012 & 0,0006 & 0,836 & * & $*$ \\
\hline$t^{*}$ pres $\left(B^{*} C\right)$ & $-23,44$ & 0,076 & $-23,44$ & 0,068 & 0,0031 & 0,310 & 0,0031 & 0,273 \\
\hline$t^{*}$ pres $\left(B^{*} D\right)$ & $-21,19$ & 0,105 & $-21,19$ & 0,095 & 0,0056 & 0,080 & 0,0056 & 0,058 \\
\hline$I^{*}$ preaq $\left(C^{*} D\right)$ & $-15,19$ & 0,234 & $-15,19$ & 0,221 & $-0,0056$ & 0,080 & $-0,0056$ & 0,058 \\
\hline$S$ & \multicolumn{2}{|c|}{48,65} & \multicolumn{2}{|c|}{47,580} & \multicolumn{2}{|c|}{1,184} & \multicolumn{2}{|c|}{1,100} \\
\hline$R^{2}$ & \multicolumn{2}{|c|}{96,07} & \multicolumn{2}{|c|}{95,700} & \multicolumn{2}{|c|}{96,570} & \multicolumn{2}{|c|}{96,300} \\
\hline$R^{2}($ adj $)$ & \multicolumn{2}{|c|}{91,83} & \multicolumn{2}{|c|}{92,200} & \multicolumn{2}{|c|}{92,870} & \multicolumn{2}{|c|}{93,800} \\
\hline$L O F$ & \multicolumn{2}{|c|}{0,29} & \multicolumn{2}{|c|}{0,319} & \multicolumn{2}{|c|}{0,202} & \multicolumn{2}{|c|}{0,268} \\
\hline Residual & \multicolumn{2}{|c|}{0,08} & \multicolumn{2}{|c|}{0,355} & \multicolumn{2}{|c|}{0,844} & \multicolumn{2}{|c|}{0,441} \\
\hline
\end{tabular}

*Termos ausentes no modelo reduzido.

$$
\begin{aligned}
& F_{M A X}= 4200,8+54,54 A+19,21 B-12,96 C+88,42 D-15,30 A^{2}-15,30 B^{2}-21,55 C^{2} \\
&+26,13 D^{2}+34,06 A D-23,44 B C-21,19 B D-15,19 C D \\
& I D=19,98+1,625 A+3,125 B+2,208 C+1,951 D+0,365 A^{2}+0,865 B^{2}+0,749 D^{2}-0,438 A C \\
& \quad+0,313 B C+0,563 B D-0,563 C D
\end{aligned}
$$

Na Equação 4 é possível verificar a função objetivo do modelo com suas respectivas restrições de indentação e de espaço experimental, garantindo que o resultado permanecerá dentro dos limites operacionais do equipamento.

$$
\left\{\begin{array}{cc}
\operatorname{Max} & F(Y)=F_{M A X} \\
& \text { St.: } \quad I D \leq 0,2 \\
& \mathbf{x}^{T} \mathbf{x} \leq \rho^{2}
\end{array}\right.
$$

Assim, obteve-se os seguintes resultados previstos para os parâmetros: Corrente de solda de $88 \%$ (5,28kA); tempo de solda de 11 ciclos; pressão dos eletrodos de 4,3 bar e; pré-aquecimento de 9 ciclos a 50\% (3kA). Diante ao intervalo de confiança de $95 \%$ dos valores ótimos, tem-se: Força máxima de cisalhamento de 4543 N (4436 a 4725 ) e indentação máxima de 0,20 mm (0,172 a 0,242). 

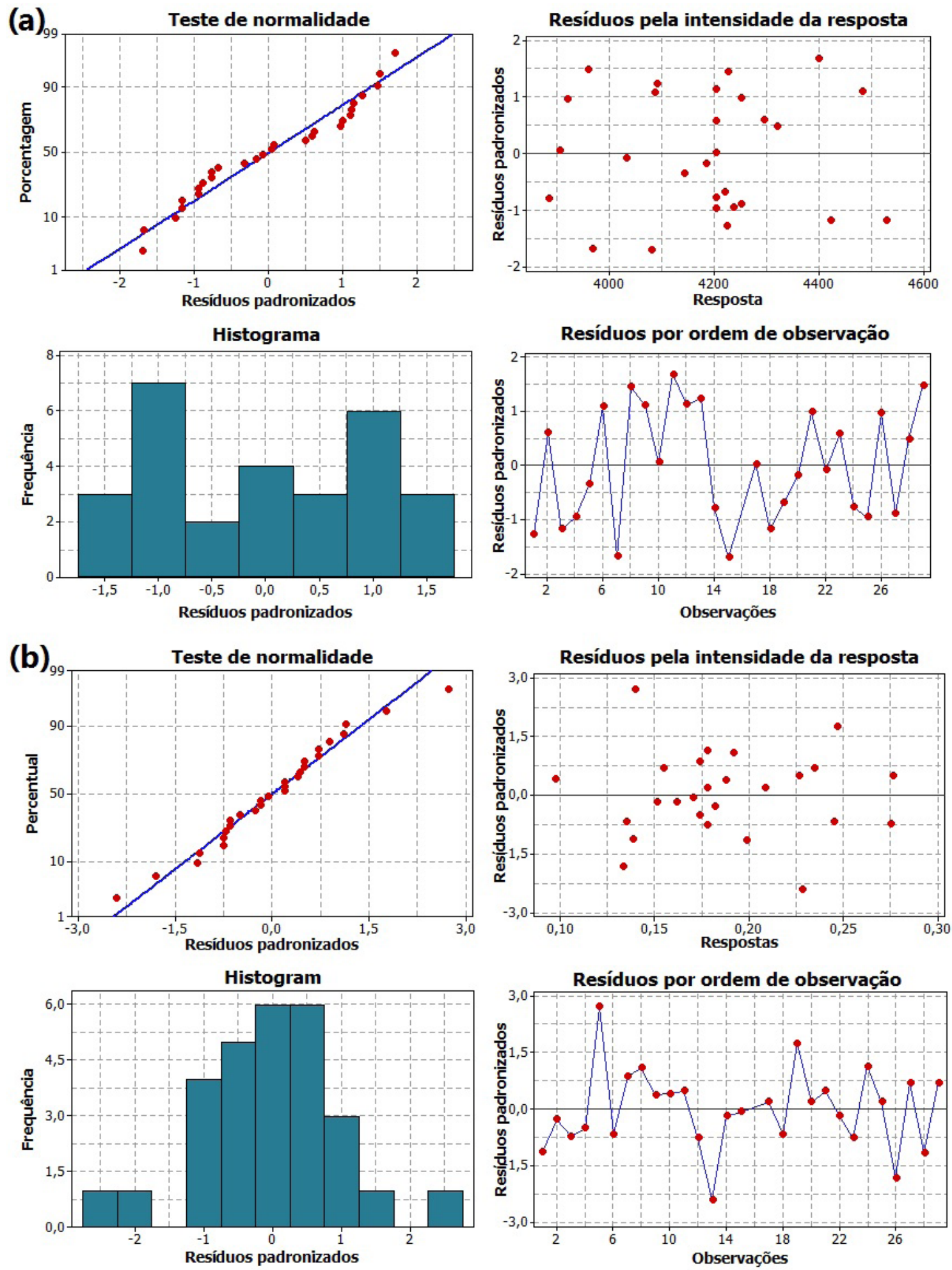

Figura 6. Análise dos resíduos: (a) força máxima de cisalhamento; (b) indentação máxima.

A partir das condições otimizadas realizou-se um ensaio de confirmação em um total de 20 amostras, como descrito na Tabela 4. Todas as amostras apresentaram modo de falha adequado, ou seja, arrancamento do ponto ou rasgamento da chapa. A média de força máxima de cisalhamento foi de $4505 \mathrm{~N}$, com um intervalo de confiança 

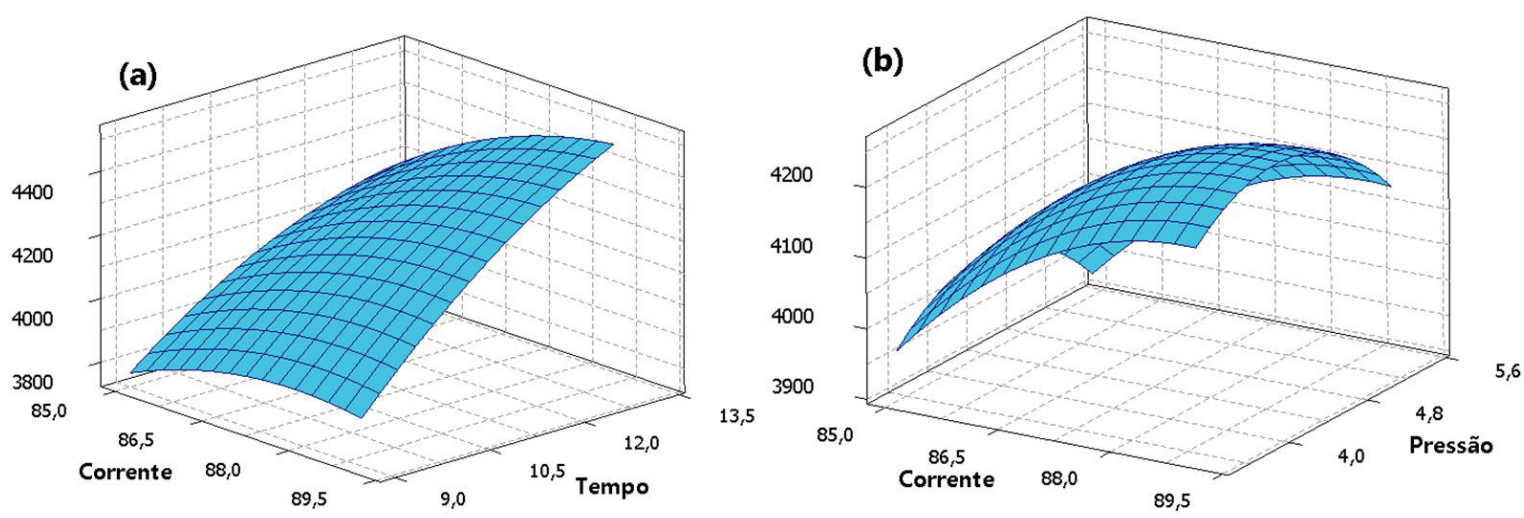

Figura 7. Superfícies de resposta para força máxima de cisalhamento. Valor fixo (a) Pressão 4,5; pré-aq 0,5 (b) Tempo 11; pré-aq 0,5 .
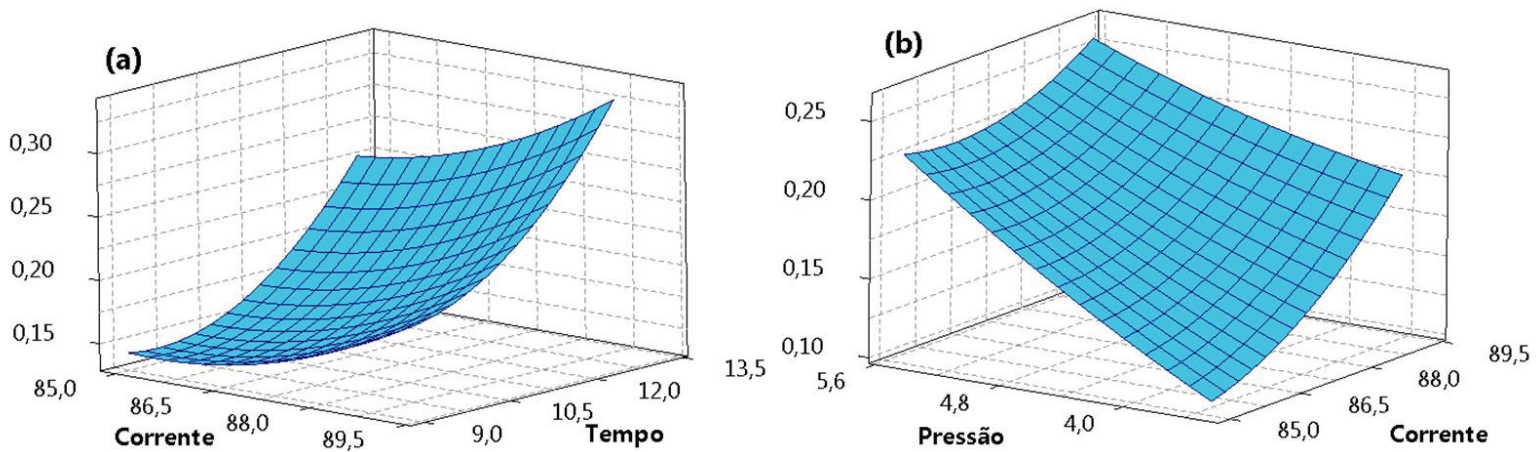

Figura 8. Superfícies de resposta para indentação máxima. Valor fixo (a) Pressão 4,5; pré-aq 0,5 (b) Tempo 11 ; pré-aq 0,5 .

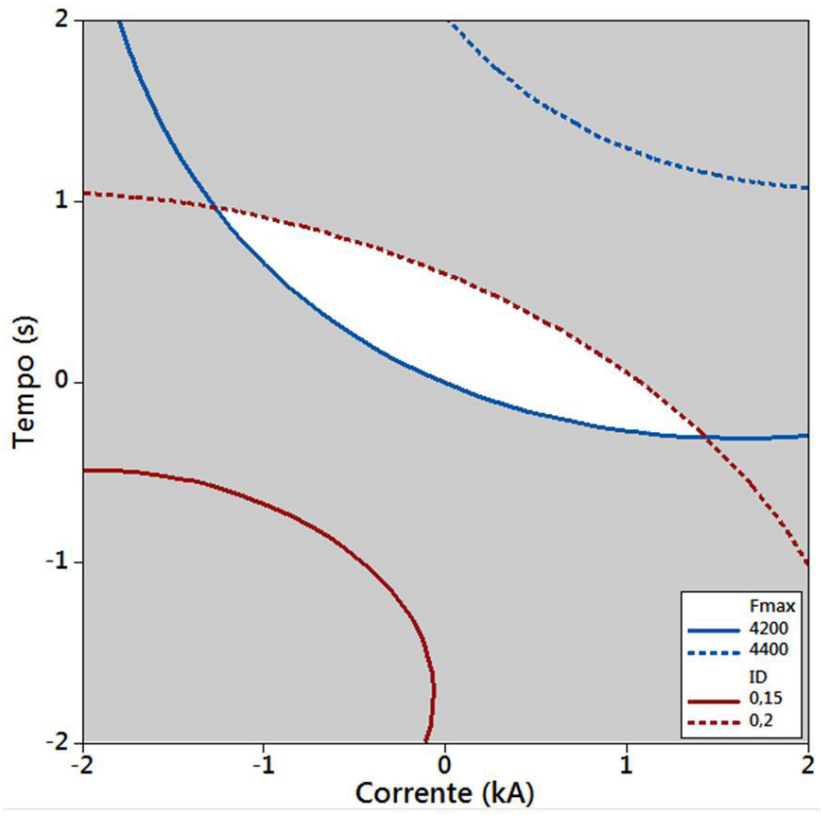

Figura 9. Região de interseção entre as curvas de força de cisalhamento e indentação. Valor fixo: Pressão 4,5; Pré-aquecimento 0,5. 
Aplicação da Metodologia de Superfície de Resposta para Otimização do Processo de Solda a Ponto no Aço Galvanizado AISI 1006

Tabela 4. Resposta dos ensaios de confirmação.

\begin{tabular}{|c|c|c|c|}
\hline Experimento & Força máx $(N)$ & Identação máx (mm) & Tipo de rompimento \\
\hline 1 & 4471 & 0,18 & rasgamento \\
\hline 2 & 4443 & 0,19 & rasgamento \\
\hline 3 & 4486 & 0,22 & rasgamento \\
\hline 4 & 4659 & 0,23 & arrancamento/rasgamento \\
\hline 5 & 4466 & 0,18 & arrancamento/rasgamento \\
\hline 6 & 4481 & 0,19 & rasgamento \\
\hline 7 & 4486 & 0,21 & arrancamento/rasgamento \\
\hline 8 & 4649 & 0,2 & rasgamento \\
\hline 9 & 4459 & 0,2 & arrancamento/rasgamento \\
\hline 10 & 4507 & 0,18 & arrancamento/rasgamento \\
\hline 11 & 4491 & 0,19 & arrancamento/rasgamento \\
\hline 12 & 4507 & 0,23 & rasgamento \\
\hline 13 & 4471 & 0,18 & rasgamento \\
\hline 14 & 4529 & 0,19 & arrancamento/rasgamento \\
\hline 15 & 4469 & 0,21 & arrancamento/rasgamento \\
\hline 16 & 4498 & 0,2 & arrancamento/rasgamento \\
\hline 17 & 4476 & 0,22 & rasgamento \\
\hline 18 & 4501 & 0,17 & arrancamento/rasgamento \\
\hline 19 & 4598 & 0,2 & rasgamento \\
\hline 20 & 4448 & 0,19 & arrancamento/rasgamento \\
\hline
\end{tabular}

de $95 \%$ entre $4444 \mathrm{~N}$ e $4566 \mathrm{~N}$. Este resultado apresenta-se dentro do intervalo desejado, de $4436 \mathrm{~N}$ à $4725 \mathrm{~N}$, totalizando um erro de $1,66 \%$ entre o valor previsto e a média dos experimentos de confirmação. A média de indentação máxima foi de 0,198 mm, com intervalo de confiança entre 0,181 mm e 0,216 mm. Esta característica da qualidade também se mostrou dentro dos limites desejados, estando entre $0,172 \mathrm{~mm}$ a 0,242 $\mathrm{mm}$, com um erro em relação a média prevista de 4,35\%.

Diante aos resultados de confirmação, gerou-se as curvas de deslocamento do eletrodo, Figura 10, com finalidade de demonstrar o comportamento da mesma a partir do momento em que é gerado calor no ponto de solda. As curvas geradas durante os experimentos ficaram condizentes a literatura, conforme [20], estando diretamente relacionada a qualidade dos pontos de solda.

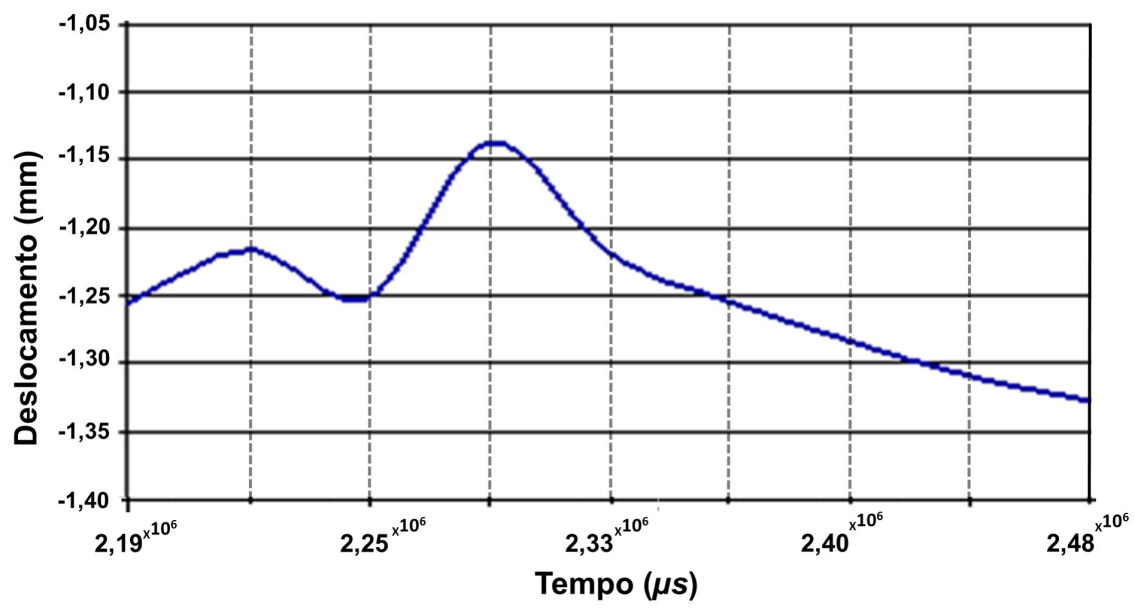

Figura 10. Curva de deslocamento do eletrodo durante a formação de um ponto de solda a partir do início da passagem de corrente e queima do revestimento. 
O deslocamento positivo no começo da curva corresponde a subida do eletrodo causada pela expansão térmica do material e pela sua mudança de estado. O ponto máximo da curva (pico superior) e a velocidade com que esse máximo é alcançado (taxa de subida), podem ser correlacionados com o grau de formação da solda, que esta diretamente relacionado a sua resistência. A taxa de descida após a corrente de soldagem ser cessada, assim como o ponto de maxima penetração do eletrodo na solda (pico inferior da curva), estão relacionados a indentação causada no ponto de solda.

Ao incluir alguns pontos durante a passagem de corrente, que antecedem a expansão térmica do material, percebeu-se um pequeno deslocamento positivo do eletrodo, causado pela queima do revestimento do material. Após esta queima do revestimento, o eletrodo volta a descer sobre a junção até o momento em que o ponto de solda começa a se formar, causando a expansão térmica e a subida do eletrodo. Este resultado confirma o que apontado por Gedeon e Eagar [4] em seu estudo. A pequena oscilação do eletrodo no momento da queima do revestimento das chapas, pode possibilitar um parâmetro extra de controle de qualidade, devido ao revestimento de zinco de aços galvanizados.

A Figura 11 mostra as curvas do deslocamento do eletrodo, iniciando no momento da pré-pressão até o momento de retorno do eletrodo, após finalizado o ponto de solda. Pode-se observar que a diferença entre a curva ideal, correspondente a um ponto de solda de qualidade, entretanto, as demais curvas, correspondem a formação de um ponto não ideal. A curva em verde representa a curva ideal que serve como referência de qualidade. A curva em amarelo ilustra uma situação de excesso de calor no ponto de solda, que compromete diretamente sua qualidade devido a um excesso de indentação. Já a curva em azul corresponde a uma situação de crescimento insuficiente do ponto de solda, comprometendo sua qualidade devido a um crescimento insuficiente do ponto de solda, o que resulta em uma baixa resistência.

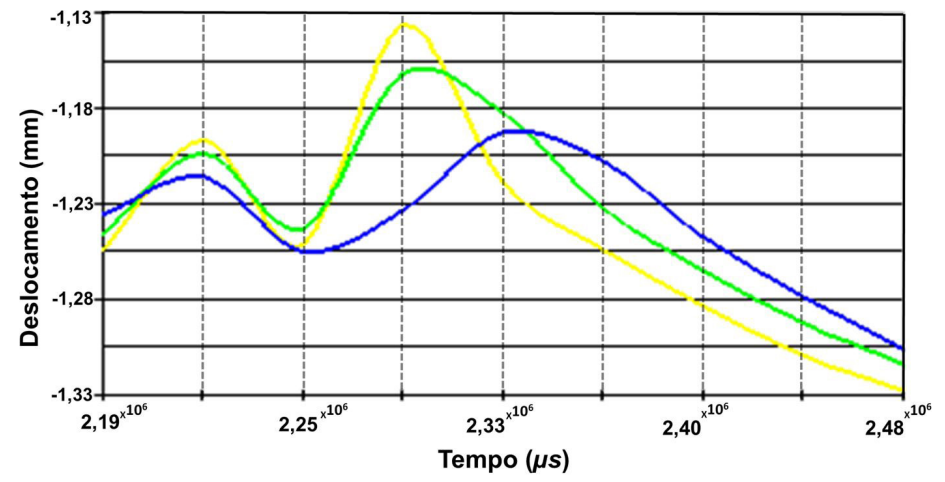

Condições de soldagem: $\square$ Ideal; $\square$ Falta de calor e; $\square$ Excesso de calor.

Figura 11. Curvas de deslocamento nas diferentes condições de soldagem incluindo a fase de queima do revestimento do material.

Analisando ainda a Figura 11, é possível observar que o primeiro pico das curvas representa um reflexo da fase de queima do revestimento. As variações na subida do eletrodo também ocorreram durante a fase de queima do revestimento, anterior a formação do ponto de solda. Este fato gera uma hipótese de que, o deslocamento do eletrodo durante a fase de queima do revestimento, pode ser útil, não somente para monitorar a sua qualidade, mas também para controlar a qualidade em tempo real, considerando que esta variação de deslocamento ocorre antes da fusão do material e com um período considerável para atuar sobre os parâmetros do processo durante a execução da soldagem, garantindo a qualidade do processo.

\section{Conclusões}

Diante a aplicação da metodologia de superfície de resposta para a otimização do processo de soldagem por pontos em aços galvanizados e, com base nos resultados obtidos nos experimentos, pode-se concluir: 
Aplicação da Metodologia de Superfície de Resposta para Otimização do Processo de Solda a Ponto no Aço Galvanizado AISI 1006

- Diante aos parâmetros investigados, a corrente $(k A)$, o tempo de solda ( $s$ ) e a pressão (bar) foram significativos, como descrito na literatura. Porém, o parâmetro de pré-aquecimento surtiu um impacto positivo no processo de soldagem das chapas galvanizadas, melhorando as características de qualidade;

- Valores elevados de corrente, com tempos de ciclo reduzidos, proporcionaram resultados mais satisfatórios ao processo, garantindo o modo de falha (rompimento) desejado, apresentando maiores níveis de força e menores níveis de indentação;

- As características da qualidade, força máxima de cisalhamento e indentação, apresentaram um nível de correlação significativa, com valor de Pearson igual à 0,657 ( $p$-value =0,000), apresentando-se dentro das condições de contorno do objeto de estudo;

- Um modelo de otimização foi proposto e verificado através dos experimentos de confirmação, garantindo um ponto de solda com resistência otimizada, apresentando valor médio de $4505 \mathrm{~N}$, modo de falha por arrancamento do ponto ou rasgamento das chapas, conforme o recomendado pela literatura. Além disso, os valores de indentação se mostraram dentro dos limites recomendados, de $20 \%$ da espessura das chapas, com média de 0,198 mm;

- A partir dos experimentos gerados com o sensor de deslocamento LVDT e o condicionador de sinais, é possível verificar que os parâmetros de qualidade do ponto de solda estão correlacionados com os parâmetros do deslocamento do eletrodo durante a soldagem, como descrito na literatura;

- As variáveis incontroláveis do processo, tais como desgaste dos eletrodos, encaixe entre as chapas e impurezas na superfície das chapas, refletem diretamente na qualidade dos pontos, alterando os parâmetros de deslocamento do eletrodo;

- Verificou-se uma oscilação na expansão térmica na curva de deslocamento do eletrodo, causada pela queima do revestimento nas chapas galvanizadas antes da fusão do metal, tornando possível considera-la como um parâmetro de controle da qualidade.

\section{Agradecimentos}

Os autores gostariam de agradecer o suporte do CNPq, CAPES e FAPEMIG.

\section{Referências}

[1] Marques PV, Modenesi PJ, Bracarense AQ. Soldagem: fundamentos e tecnologia. Belo Horizonte: UFMG; 2009. p. 293-306.

[2] Paes MTP, Guimarães AS, Rebello JMA. Significância da expulsão e do tipo de fratura em soldagem por pontos. Soldagem e Materiais. 1989;1(2):51-57.

[3] Pereira MA, Ferreira JM, Loureiro A, Costa JDM, Bártolo PJ. Effect of process parameters on the strength of resistance spot welds in 6082-T6 aluminum alloy. Materials \& Design. 2010;31(5):24542463. http://dx.doi.org/10.1016/j.matdes.2009.11.052.

[4] Gedeon SA, Eagar TW. Resistance spot welding of galvanized steel: part II. Mechanisms of spot weld nugget formation. Metallurgical Transactions. B, Process Metallurgy. 1986;17B(4):887-901. http://dx.doi.org/10.1007/BF02657152.

[5] Aslanlar S. The effect of nucleus size on mechanical properties in electrical resistance spot welding of sheets used in automotive industry. Materials \& Design. 2006;27(2):125-131. http://dx.doi. org/10.1016/j.matdes.2004.09.025.

[6] Goodarzi M, Marashi PS, Pouranvari M. Dependence of overload performance on weld attributes for resistance spot welded galvanized low carbon steel. Journal of Materials Processing Technology. 2009;209(9):4379-4384. http://dx.doi.org/10.1016/j. jmatprotec.2008.11.017.
[7] Almeida FA, Gomes GF, Paula VR, Corrêa JE, Paiva AP, Gomes $\mathrm{JHF}$, et al. A weighted mean square error approach to the robust optimization of the surface roughness in an AISI 12L14 free-machining steel-turning process. Journal of Mechanical Engineering. 2018;64(3):147-156.

[8] Naves FL, Paula TI, Balestrassi PP, Braga WLM, Sawhney RS, Paiva AP. Multivariate normal boundary intersection based on rotated factor scores: a multiobjective optimization method for methyl orange treatment. Journal of Cleaner Production. 2017;143:413439. http://dx.doi.org/10.1016/j.jclepro.2016.12.092.

[9] Tak BY, Tak BS, Kim YJ, Park YJ, Yoon YH, Min GH. Optimization of color and COD removal from livestock wastewater by electrocoagulation process: application of Box-Behnken design (BBD). Journal of Industrial and Engineering Chemistry. 2015;28:307-315. http://dx.doi.org/10.1016/j.jiec.2015.03.008.

[10] Pi K, Xia M, Yang X, Wu P, Chen S, Yang M, et al. Optimization of COD decrease from tobacco wastewater by $\mathrm{Ca} / \mathrm{Mg} / \mathrm{Al}$ coagulant using RSM. Journal of Water Process Engineering. 2015;5:166171. http://dx.doi.org/10.1016/j.jwpe.2014.11.002.

[11] Sheydaei M, Aber S, Khataee A. Preparation of a novel $\gamma$-FeOOH-GAC nano composite for decolorization of textile wastewater by photo Fenton-like process in a continuous reactor. Journal of Molecular Catalysis A Chemical. 2014;392:229-234. http://dx.doi.org/10.1016/j.molcata.2014.05.019. 
[12] Anouzla A, Abrouki Y, Souabi S, Safi M, Rhbal H. Colour and COD removal of disperse dye solution by a novel coagulant: application of statistical design for the optimization and regression analysis. Journal of Hazardous Materials. 2009;166(2-3):1302-1306. http:// dx.doi.org/10.1016/j.jhazmat.2008.12.039. PMid:19168285.

[13] Montgomery DC. Design and analysis of experiments. 8th ed. New York: John Wiley \& Sons; 2013.

[14] Rowlands H, Antony J. Application of design of experiments to a spot welding process. Assembly Automation. 2003;23(3):273279. http://dx.doi.org/10.1108/01445150310486549.

[15] Martín O, Lopez M, Martín F. - Artificial neural networks for quality control by ultrasonic testing in resistance spot welding. Journal of Materials Processing Technology. 2007;183(2-3):226233. http://dx.doi.org/10.1016/j.jmatprotec.2006.10.011.

[16] Liang C, Liu X. Strength prediction of sheet to tube single sided resistance spot welding. Materials \& Design. 2009;30(10):43284334. http://dx.doi.org/10.1016/j.matdes.2009.04.015.

[17] Lin HL, Chou T, Chou C-P. Optimization of resistance spot welding process using Taguchi method and a neural network. Experimental Techniques. 2007;31(5):30-36. http://dx.doi. org/10.1111/j.1747-1567.2007.00186.x.

[18] Cho Y, Li W, Hu SJ. Design of experiment analysis and weld lobe estimation for aluminum resistance spot welding. Welding Journal. 2006;85(3):45-51.

[19] Darwish SM, Al-Dekhial SD. Micro-hardness of spot welded (BS 1050) commercial aluminium as correlated with welding variables and strength attributes. Journal of Materials Processing Technology. 1999;91(1-3):43-51. http://dx.doi.org/10.1016/ S0924-0136(98)00414-2.

[20] Jou M. Real time monitoring weld quality of resistance spot welding for the fabrication of sheet metal assemblies. Journal of Materials Processing Technology. 2003;132(1-3):102-113. http://dx.doi.org/10.1016/S0924-0136(02)00409-0.

[21] Manladan SM, Yusof F, Ramesh S, Zhang Y, Luo Z, Ling Z. Microstructure and mechanical properties of resistance spot welded in welding-brazing mode and resistance element welded magnesium alloy/austenitic stainless steel joints. Journal of Materials Processing Technology. 2017;250:45-54. http:// dx.doi.org/10.1016/j.jmatprotec.2017.07.006.

[22] Shan H, Zhang Y, LiY, Luo Z. Dissimilar joining of AZ31B magnesium alloy and pure copper via thermo-compensated resistance spot welding. Journal of Manufacturing Processes. 2017;30:570-581. http://dx.doi.org/10.1016/j.jmapro.2017.10.022.
[23] Chen N, Wang HP, Carlson BE, Sigler DR, Wang M. Fracture mechanisms of $\mathrm{Al} /$ steel resistance spot welds in lap shear test. Journal of Materials Processing Technology. 2017;243:347-354. http://dx.doi.org/10.1016/j.jmatprotec.2016.12.015.

[24] Martín Ó, De Tiedra P, San-Juan M. Combined effect of resistance spot welding and precipitation hardening on tensile shear load bearing capacity of A286 superalloy. Materials Science and Engineering A. 2017;688:309-314. http://dx.doi.org/10.1016/j. msea.2017.02.015.

[25] Feng Y, Li Y, Luo Z, Ling Z, Wang Z. Resistance spot welding of Mg to electro-galvanized steel with hot-dip galvanized steel interlayer. Journal of Materials Processing Technology. 2016;236:114-122. http://dx.doi.org/10.1016/j.jmatprotec.2016.05.015.

[26] Pouranvari M, Marashi P, Goodarzi M. Failure mode of dissimilar resistance of spot welds between austenitic stainless and low carbon steels. In: Proceedings of Metal; 2008; Czech Republic. Czech Republic: Tanger; 2008. p. 1-6.

[27] Wan X, Wang Y, Zhao D, Huang Y, Yin Z. Weld quality monitoring research in small scale resistance spot welding by dynamic resistance and neural network. Measurement. 2017;99:120 127. http://dx.doi.org/10.1016/j.measurement.2016.12.010.

[28] Singh D, Rao PV. A surface roughness prediction model for hard turning process. International Journal of Advanced Manufacturing Technology. 2007;32(11-12):1115-1124. http:// dx.doi.org/10.1007/s00170-006-0429-2.

[29] American Welding Society. AWS/SAE D8.9M: recommended practices for test methods for evaluating the resistance spot welding behavior of automotive sheet steel materials. Miami: AWS; 2002.

[30] Wan Z, Wang HP, Chen N, Wang M, Carlson BE. Characterization of intermetallic compound at the interfaces of Al-steel resistance spot welds. Journal of Materials Processing Technology. 2017;242:1223. http://dx.doi.org/10.1016/j.jmatprotec.2016.11.017.

[31] Zhang Y, Shan H, Li Y, Guo J, Luo Z, Ma CY. Joining aluminum alloy 5052 sheets via novel hybrid resistance spot clinching process. Materials \& Design. 2017;118:36-43. http://dx.doi. org/10.1016/j.matdes.2017.01.017.

[32] Pouranvari M. Fracture toughness of martensitic stainless stee resistance spot welds. Materials Science and Engineering A. 2017;680:97-107. http://dx.doi.org/10.1016/j.msea.2016.10.088.

[33] Ighodaro OL, Biro E, Zhou YN. Comparative effects of Al-Si and galvannealed coatings on the properties of resistance spot welded hot stamping steel joints. Journal of Materials Processing Technology. 2016;236:64-72. http://dx.doi.org/10.1016/j. jmatprotec.2016.03.021. 